> L'atteinte rénale est l'une des manifestations les plus graves de la maladie de Fabry. Nous rapportons ici l'expérience de l'enzymopathie substitutive avec l'agalsidase $\beta$ chez neuf patients atteints de maladie de Fabry. Un suivi sur 4 ans a été réalisé avec une surveillance particulière de l'évolution rénale et cardiaque. <

L'atteinte rénale est une des manifestations les plus graves de la maladie de Fabry puisqu'elle conduit les patients

\section{Enzymothérapie substitutive chez neuf patients atteints de la maladie de Fabry}

Éric Alamartine

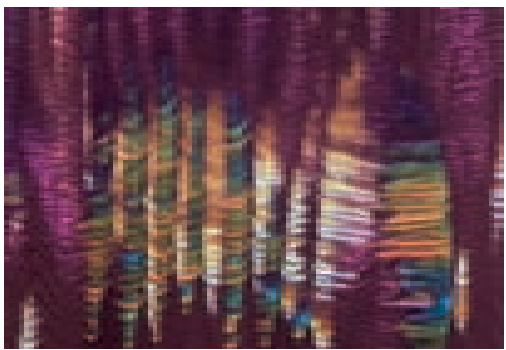

Service de Néphrologie-DialyseTransplantation Rénale, Hôpital Nord, 42055 Saint-Étienne Cedex2, France. eric.alamartine@ chu-st-etienne.fr en dialyse ou transplantation rénale. La néphropathie apparaît chez l'adulte jeune avec une protéinurie croissante. Dès lors, l'évolution vers l'insuffisance rénale est certaine. Elle est terminale vers l'âge de 40 ans. Histologiquement, la vacuolisation des cellules épithéliales podocytaires est caractéristique mais d'autres cellules, mésangiales, endothéliales et tubulaires sont atteintes. Les dépôts sont visualisés au mieux par le bleu de toluidine. L'évolution rénale est probablement le meilleur moyen d'apprécier l'efficacité du traitement enzymatique substitutif, car il s'agit d'un paramètre dur (mort rénale) et évaluable dans un délai raisonnable.

Nous rapportons l'expérience de l'enzymothérapie substitutive avec l'agalsidase $\beta$ chez neuf patients traités dans notre centre depuis 4 ans avec une orientation toute particulière sur l'évolution des fonctions vitales, rénales et cardiaques.

\section{Méthodes}

Dans tous les cas le diagnostic de maladie de Fabry a été étayé par un dosage de l' $\alpha$-galactosidase A et le génotypage. Quatre des neuf patients avaient une néphropathie patente et deux autres avaient déjà bénéficiés d'une greffe rénale lorsque le traitement a débuté. Ces deux patients seront considérés à part dans l'analyse.

L'évaluation a porté sur les points suivants:

1. Tolérance au traitement substitutif par enregistrement des effets secondaires et recherche régulière des anticorps anti-agalsidase $\beta$. II a été réalisé un test ELISA de dépistage suivi d'un test de confirmation en radio-immuno-précipitation en cas de positivité (Genzyme, Cambridge, États-Unis).

2. Évolution rénale déterminée par la mesure séquentielle de la protéinurie des 24 heures, de la créatinine plasmatique et du débit de filtration glomérulaire mesuré par clairance de l'inuline. Nous disposons d'une biopsie rénale chez cinq patients, mais seul, un malade a eu un contrôle après traitement.

3. Évolution cardiaque par mesure de l'indice de masse ventriculaire gauche en échographie trans-thoracique, complétée en cas de myocardiopathie d'un test d'effort, d'une $\mathrm{VO}_{2}$ max et d'un holter $\varepsilon C G$.

4. Mesure du globotriaosylcéramide (GL-3) plasmatique (réalisé par le laboratoire Genzyme Corporation-Clinical Specialty Laboratory, Framingham, États-Unis).

\section{Suivi du traitement}

Nous avons utilisé, pour les neuf patients, l'agalsidase $\beta$ (Fabrazyme $^{\circledast}$ ) [1]. La durée d'administration était toujours de 4 heures au début, progressivement et régulièrement raccourcie ensuite. Initialement délivrée à la posologie recommandée de $1 \mathrm{mg} / \mathrm{kg} / \mathrm{l} 4$ jours, la dose d'agalsidase $\beta$ a été augmentée afin de ne pas gaspiller de produit du fait du conditionnement en flacons de $35 \mathrm{mg}$. La majorité des patients reçoivent actuellement $1,5 \mathrm{mg} / \mathrm{kg} / 14$ jours. Le recul est de 4 ans pour sept patients, 1 an pour deux patients. Les résultats donnés sont les valeurs avant l'initiation du traitement (Tableau I) et les valeurs au dernier point de suivi. 
Le traitement symptomatique par carbamazépine était poursuivi à la convenance personnelle des patients. En revanche, nous avons systématiquement introduit un inhibiteur de l'enzyme de conversion en cas de protéinurie, d'insuffisance rénale ou d'hypertension artérielle.

\section{Complications}

Le problème essentiel fut l'apparition, au bout du $3^{e}$ mois de traitement, de réactions d'intolérance en cours de perfusion, avec essentiellement des manifestations allergiques : prurit, rash cutané, picotement laryngé. Cette intolérance clinique correspondait à l'augmentation du débit de perfusion et s'amendait avec le ralentissement de celle-ci ainsi qu'avec l'emploi d'une prémédication corticoïdes et anti-Hl. Au long cours, deux patients se plaignent d'une fatigue après la perfusion. La tolérance s'est révélée globalement plutôt bonne et nous avons pu poursuivre le traitement à domicile chez quatre patients.

L'apparition d'anticorps anti-agalsidase $\beta$ fut retrouvée chez $80 \%$ des patients, mais seulement $40 \%$ de ceux-ci avaient des anticorps significatifs, positifs en radio-immuno-précipitation.

\section{Évolution de la néphropathie}

Les malades de notre série avaient une néphropathie peu protéinurique. Dans un seul cas, nous avons noté une protéinurie de l'ordre de $1,5 \mathrm{~g} / 24 \mathrm{~h}$. Chez les autres, le taux de protéinurie n'excédait pas $0,5 \mathrm{~g} / 24 \mathrm{~h}$. Hormis ce cas de forte protéinurie qui fut réduite à $0,5 \mathrm{~g} / 24 \mathrm{~h}$, les taux sont restés à peu près inchangés. La créatininémie est passée de $77 \pm 11$ à $71 \pm 21 \mu \mathrm{mol} / \mathrm{I}$ (NS). La clairance de l'inuline (Tableau II) est passée de $94 \pm 21$ à $96 \pm 26 \mathrm{ml} / \mathrm{min} / 1,73 \mathrm{~m}^{2}$ (NS). Une biopsie rénale a pu être renouvelée chez un patient. Les dépôts de GL-3 étaient toujours présents dans les cellules podocytaires mais avaient disparu des cellules endothéliales. À noter l'apparition, chez ce malade, de kystes parapyéliques, alors que l'évolution fonctionnelle et histologique était favorable.

La description de l'atteinte rénale chez les deux greffés rénaux est plus délicate du fait de facteurs confondants évidents (néphrotoxicité médicamenteuse, néphropathie chronique de l'allogreffe...). La tolérance a été remarquable. La clairance de l'inuline est passée de 27 à $23 \mathrm{ml} / \mathrm{min} / 1,73 \mathrm{~m}^{2}$ chez l'un et de 29 à $33 \mathrm{ml} /$ $\min / 1,73 \mathrm{~m}^{2}$ chez l'autre, alors qu'aucun événement significatif n'a été noté.

\section{Action sur le myocarde}

Aucune myocardiopathie n'est apparue chez les patients qui n'en présentaient pas au début de l'étude. Cinq malades avaient une myocardiopathie hypertrophique, dont quatre très sévères avec des IMVG de 288, 300, 333 et $555 \mathrm{~g} / \mathrm{m}^{2}$ respectivement. Aucune amélioration significative de la myocardiopathie n'a été constatée chez eux. Une aggravation impressionnante de 333 à $551 \mathrm{~g} / \mathrm{m}^{2}$ a même été notée dans un cas. Ce sont les symptômes cardiaques qui sont le plus mal ressentis. Les patients avec une importante hypertrophie ventriculaire gauche se plaignant beaucoup de palpitations et de douleurs thoraciques.

\begin{tabular}{|c|c|c|c|c|c|c|c|c|c|}
\hline Patient & Sexe & Âge & Enzyme & Mutation & Rein & $\begin{array}{l}\text { Protéinurie } \\
\text { g/24h }\end{array}$ & $\begin{array}{l}\text { Créatinine } \\
\mu \mathrm{mol} / \mathrm{I}\end{array}$ & $\begin{array}{l}\text { DFG } \\
\mathrm{ml} / \mathrm{min} / 1,73 \mathrm{~m}^{2}\end{array}$ & Autres atteintes \\
\hline $\mathrm{CL}$ & $\mathrm{F}$ & 17 ans & $20 \%$ & D313y \& G411D & - & 0 & 69 & 110 & \\
\hline$\varepsilon G$ & $\mathrm{H}$ & 19 ans & $0 \%$ & N224D & - & 0 & 67 & 122 & \\
\hline SS & $\mathrm{H}$ & 41 ans & $0 \%$ & L300P & - & 0 & 93 & 146 & myocardiopathie \\
\hline$\varepsilon P$ & $\mathrm{H}$ & 43 ans & $0 \%$ & $R 227 X$ & Néphropathie & 0,48 & 93 & 80 & \\
\hline PV & $H$ & 45 ans & $0 \%$ & $89 \mathrm{delG}$ & Néphropathie & 0,70 & 102 & 65 & myocardiopathie \\
\hline MG & $\mathrm{F}$ & 58 ans & $29 \%$ & N224D & Néphropathie & 0,45 & 81 & 71 & myocardiopathie \\
\hline$\varepsilon B$ & $F$ & 57 ans & $4 \%$ & $\mathrm{R} 227 \mathrm{X}$ & Néphropathie & 1,50 & 71 & 86 & myocardiopathie \\
\hline$J \mathrm{~L}$ & $\mathrm{H}$ & 50 ans & $0 \%$ & D313y \& G411D & Greffe & 0,49 & 244 & 27 & $\begin{array}{l}\text { myocardiopathie } \\
+ \text { AVC }\end{array}$ \\
\hline$J B$ & $\mathrm{~F}$ & 56 ans & $50 \%$ & délétion & Greffe & 0 & 199 & 29 & $\begin{array}{l}\text { insuffisance } \\
\text { respiratoire }\end{array}$ \\
\hline
\end{tabular}

Tableau I. Caractéristiques des patients et données avant traitement enzymatique. 
Le GL-3 plasmatique a pu être comparé chez les sept patients pour lesquels nous avons 4 ans de recul. II a diminué de $7,3 \pm 4,3$ à $3,9 \pm 1,3 \mu \mathrm{mol} / \mathrm{l} \quad(p=0,018)$ (Figure 1).

\section{Discussion}

Nous avons effectué un suivi sur 4 ans de neuf patients avec évaluation des paramètres biologiques en particulier ceux ce la fonction rénale

Quatre de ces personnes sont des femmes, dont trois ont une forme grave de la maladie. Cela souligne, si besoin en était encore, le risque évolutif chez les femmes.

Sur le plan rénal, les patients étaient relativement hétérogènes avant l'instauration du traitement enzymatique puisque trois d'entre eux n'avaient pas de néphropathie patente, quatre avaient une néphropathie débutante et deux avaient subi une transplantation. Chez les cinq malades non greffés qui ont été évalués après 4 ans de traitement, on note une stabilité du débit de filtration glomérulaire étudié par la clairance de l'inuline, marqueur de référence. Ce résultat apparemment nul est en fait favorable, car l'histoire naturelle de la néphropathie est beaucoup plus sévère. $\varepsilon$ n général, le stade terminal de l'insuffisance rénale est atteint 10 ans après l'apparition de la protéinurie, soit une perte de la filtration glomérulaire de $10 \mathrm{ml} / \mathrm{mn}$ par an. L'évolution du taux de la protéinurie nous semble un critère peu pertinent à court terme, car la protéinurie est en général faible et l'emploi des IEC masque encore plus l'évolution. Du fait de cet aspect quantitatif de la protéinurie, il s'agit d'une maladie où la recherche d'une microalbuminurie devrait être utilisée très largement. Nous ne pouvons qu'insister sur la nécessité d'une prise en charge complète de la néphropathie. La biopsie

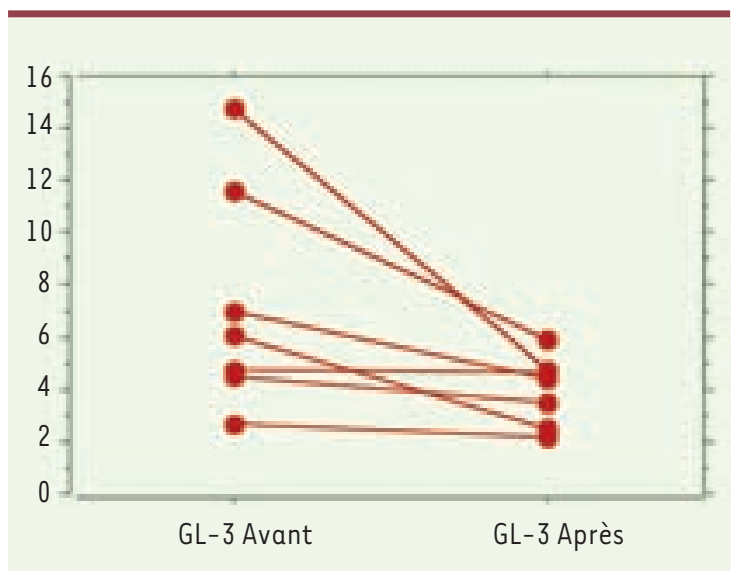

Figure 1. Taux plasmatiques de GL-3 ( $\mu$ mol/l). rénale est d'une grande utilité: elle a permis le diagnostic de cas difficiles, elle quantifie et détaille les lésions. Un traitement néphroprotecteur optimisé est indispensable dans cette prise en charge néphrologique [2]. Signalons l'apparition de kystes sinusaux sous traitement. Cette manifestation rénale peu connue semble survenir fréquemment [3]. À noter que nous avons traité deux patients greffés rénaux, avec l'objectif d'une protection des autres organes. Notre expérience confirme la parfaite faisabilité de l'enzymothérapie chez les greffés déjà démontrée dans une étude récente [4]. Ce point est d'autant plus important que les patients atteints de maladie de Fabry ont de bons résultats en greffe de rein [5]. Pour mémoire, rappelons que si la maladie de Fabry ne récidive par sur le greffon, le greffon ne corrige pas le déficit enzymatique du receveur. En somme, le bénéfice rénal que nous avons observé avec l'agalsidase $\beta$ est en accord avec les données actuellement publiées, qu'il s'agisse de cas cliniques [6] ou d'études multicentriques [7].

L'autre critère favorable, en terme d'efficacité du traitement enzymatique, est la baisse significative du GL-3 plasmatique. Certes, il s'agit d'un critère «intermédiaire », mais dont la signification clinique est forte. L'utilité du dosage du GL-3 plasmatique serait toutefois limitée chez les patients atteints de mutations particulières [8]. Comme nous l'avons déjà mentionné plus haut, nous n'avons pas noté d'évolution favorable sur la myocardiopathie. Les hypertrophies ventriculaires gauches étaient il est vrai déjà considérables à l'instauration du traitement.

Toutes ces données sont en faveur de l'instauration précoce du traitement enzymatique substitutif. II semble qu'il y ait un point de non-retour, notamment en ce qui concerne les atteintes rénales et cardiaques. C'est avant la constitution de lésions histologiques plus ou moins irréversibles qu'il semble logique de restaurer l'activité enzymatique [9]. Rappelons que, sur le plan rénal, on peut attendre une élimination du GL-3 des cellules endothéliales à turn-over rapide, mais non des cellules podocytaires qui ne se renouvellent pas. De plus, les lésions non spécifiques, secondaires à l'ischémie causée par l'obstruction endothéliale, sont à la fois précoces et irréversibles.

\begin{tabular}{|lll}
\hline Patient & DFG avant $\left(\mathrm{ml} / \mathrm{min} / 1,73 \mathrm{~m}^{2}\right)$ & DFG après $\left(\mathrm{ml} / \mathrm{min} / 1,73 \mathrm{~m}^{2}\right)$ \\
\hline CL & 110 & 123 \\
\hline EG & 122 & 121 \\
\hline SS & 146 & \\
\hline EP & 80 & 95 \\
\hline PV & 65 & \\
\hline MG & 71 & 61 \\
\hline EB & 86 & 81 \\
\hline JL & 27 & 23 \\
\hline JB & 29 & 33 \\
\hline
\end{tabular}

Tableau II. Évolution du débit de filtration glomérulaire (clairance de l'inuline). 
La dernière question que pose notre petite cohorte est celle de la signification des anticorps anti-agalsidase $\beta$. Comme dans les études princeps, l'apparition de ceux-ci n'ayant en rien modifié le bénéfice observé, on peut conclure que ces anticorps ne sont pas neutralisants. Toutefois, la question reste en suspens du fait de l'absence d'amélioration objective de certaines données, d'autant qu'un travail récent a remis en doute l'innocuité de ces anticorps [10].

\section{Conclusion}

Notre expérience de 4 années d'enzymothérapie substitutive chez neuf malades a montré un bénéfice net pour l'atteinte rénale, beaucoup plus incertain pour l'atteinte cardiaque. II semble que le bénéfice de l'enzymothérapie substitutive soit fonction de la précocité du traitement. $\diamond$

\section{SUMMARY}

Enzyme replacement therapy in nine patients with Fabry disease Kidney involvement is a major complication of Fabry disease. Here, we report the experience of treatment by enzyme replacement with agalsidase $\beta$ in 9 patients with Fabry disease. A 4 years follow up was performed with a particular survey of renal and cardiac evolution. $\Delta$

\section{RÉFÉRENCES}

1. Eng CM, Guffon N, Wilcox WR, et al. Safety and efficacy of recombinant human alpha galactosidase A replacement therapy in Fabry's disease. N Engl J Med 2001 ; $345: 9-16$.

2. Warnock DG. Fabry disease: diagnosis and management, with emphasis on the renal manifestations. Curr Opin Nephrol Hypertens $2004 ; 14: 87$ 95.

3. Ries M, Bove Bettis KE, Choyke P, et al. Parapelvic kidney cysts : a distinguishing feature with high prevalence in Fabry disease. Kidney Int $2004 ; 66: 978-82$.

4. Mignani R, Panichi V, Giudicissi A, et al. Enzyme replacement therapy with agalsidase beta in kidney transplant patients with Fabry disease : a pilot study. Kidney Int 2004 ; 65 : 1381-5.

5. 0jo A, Meier-Kriesche HU, Friedman G, et al. Excellent outcome of renal transplantation in patients with Fabry's disease. Transplantation 2000 ; 69: 2337-9.

6. De Schoemakere G, Chauveau D, Grünfeld JP. Enzyme replacement therapy in Anderson-Fabry's disease : beneficial clinical effect on vital organ function. Nephrol Dial Transplant 2003; 18 : 33-5.

7. Wilcox WR, Banikazemi M, Guffon N, et al. Long-term safety and efficacy of enzyme replacement therapy for Fabry disease. Am J Hum Genet 2004 ; $75: 65-74$.

8. Young $\varepsilon$, Milks K, Morris $P$, et al. Is globotriaosylceramide a useful marker in Fabry disease? Acta Paediatr 2005 ; 94 (suppl) : 51-4.

9. Siamopoulos KC. Fabry disease : kidney involvement and enzyme replacement therapy. Kidney Int $2004 ; 65: 744-53$.

10. Lindthorst GL, Hollak CEM, Donker-Koopman $\varepsilon$, et al. Enzyme therapy for Fabry disease : neutralizing antibodies toward agalsidase alpha and beta. Kidney Int 2004 ; 66 : 1589-95.

\section{TIRÉS À PART}

$\varepsilon$. Alamartine 